JOURNAL DE PHYSIQUE

Colloque $C 1$. supplément au $n^{\circ} 2$, Tome 47, février 1986

page $\mathrm{C} 1-871$

\title{
DEFECT STRUCTURE AND PHYSICAL PROPERTIES OF STRONTIUM TITANATE
}

\author{
Y. DANSUI, B. CALES and F. GERVAIS \\ Centre de Recherches sur la Physique des Hautes Températures, \\ C.N.R.S., F-45045 orléans Cedex, France
}

\begin{abstract}
Résumé - L'étude des propriétés physiques du titanate de strontium dopé au niobium a été menée sur des échantillons polycristallins. Les propriétés électriques d'échantillons rêduits ont été analysées par mesure de la conductivité et du pouvoir thermoélectrique. Des spectres IR ont été obtenus sur des échantillons polis optiquement, montrant ainsi les possibilités offertes par 1 'emplois de matériaux polycristallins dans ce domaine.

Abstract - The physical properties of Nb-doped strontium titanate have been studied on polycrystalline samples. The electrical properties of reduced specimens have been analyzed by measurement of the conductivity and the thermoelectric nower. IR reflection spectra have been obtained on optically polished samples. This result shows the potential interest of polycrystalline specimens for optical studies on such materials.
\end{abstract}

\section{I - INTRODUCTION}

In the last few years, various studies have been devoted to the transport properties, the ferroelectric properties or the temperature dependence of the dielectric constant of strontium titanate /1-3/. More recently, some preliminary experiments carried out on single crystals of $\mathrm{Nb}$-doped strontium titanate ( 1 to $2 \mathrm{~mol} \% \mathrm{Nb}_{2} \mathrm{O}_{5}$ ) /4/ have clearly revealed the deep modifications of the lattice dynamic and electronic properties induced by the doping. The electron-phonon coupling is then high enough to make the material superconductor at temperatures lower than $0.7 \mathrm{~K}$. It thus appeared attractive to perform a thoroughly study of these properties that appear to be specific of strontium titanate.

However, owing to the difficulties of material supply, the present work has been achieved by using polycrystalline samples instead of single crystals, even for optical measurements. This paper reports on electrical conductivity and Seebeck coefficient measurements and on the infrared reflection spectrum of $\mathrm{Nb}$-doped strontium titanate $\left(0.5\right.$ mol $\left.\% \mathrm{Nb}_{2} \mathrm{O}_{5}\right)$.

\section{II - EXPERIMENTAL PROCEDURE}

A fine strontium titanate powder doped with $0.5 \mathrm{~mol} \%$ of niobium oxide has been synthetized according to an organometallic route derived from the processing reported by Pechini /5/. A titanium solution was first preapared by dissolution of tetraisopropyl orthotitanate into a mixture of ethylene glycol and citric acid. During esterification on heating, titanium ions are chelated by the ester while an exhaust of isopropyl alcohol is noted. The titanium oxide content of this solution is obtained after polymerisation of the ester and ignition. An appropriate quantity of strontium ions are then introduced in the titanium solution via strontium carbonate dissolved into formic acid. Doping is achieved by the addition of niobium-V ethoxide. After homogeneization, the mixture is allowed to polymerize to an amorphous resin that was ignited at $900 \mathrm{~K}$ to yield a fine $\mathrm{vb}$-doped strontium titanate powder.

Analysis by TEM and X-ray line broadening measurements indicate a mean crystallite 
size of about $250 \AA$ (Fig. 1). Sintered samples have been obtained after firing isostatically compacted samples. The density of the specimens increaIses rapidly, with the sintering temperature and a maximum density ( $>99.5 \%$ of the theoretical density estimated to 5.12) is reached at $1700 \mathrm{~K}$.

Specimens for electrical measurements have been cut into rods using diamond tools while those for IR investigations have been optically polished. They were then reduced at $1300 \mathrm{~K}$ under various oxygen partial pressures and quenched in order to fix the stoichiometric composition to given values.

Sample A was obtained for an oxygen partial pressure of

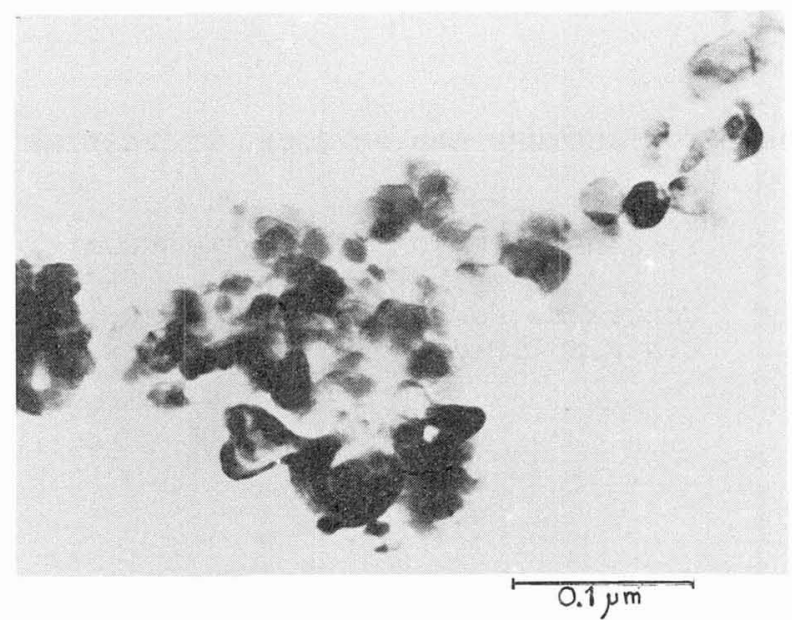

Fig. 1 - TEM micrograph of the $\mathrm{Nb}^{-\mathrm{SrTiO}_{3}}$ powder $\mathrm{pO}_{2}=10^{-11} \mathrm{MPa}$ while sample $\mathrm{B}$ was reduced under $\mathrm{pO}_{2}=10^{-18} \mathrm{MPa}$. The infrared reflectivity was measured with the aid of a BRUKER ET IR spectrometer on optically polished specimens that do not exhibit significant diffusion at least above $10 \mu \mathrm{m}$.

\section{III - ELECTRICAL PROPERTIES}

The dc conductivity of reduced $\mathrm{Nb}$-doped strontium titanate was measured, using a classical four-probe technique, in a large temperature range $(78-1300 \mathrm{~K})$. The variation of the conductivity versus temperature is reported in Fig. 2 for different stoichiometric compositions of the $0.5 \mathrm{mo} 1 \% \mathrm{Nb}$-doped sample. They are in good agreement with the results previous$1 y$ reported in the literature for the electrical conductivity in strontium titanate $12,3 /$. Two different regimes may be distinguished in Fig. 2. The first one corresponds to temperatures highex than about $300 \mathrm{~K}$ in the case of sample $A$ or higher than $200 \mathrm{~K}$ only for sample $B$. In this regime the two samples are characterized by a meta1lic-type conductivity that slowly decreases as the temperature increa ses. As reported by Tufte and Chapman / $3 /$ or Frederikse et al $/ 2 /$, the niobium ions forms shallow donor centers and may be considered as hydrogen-like donors. Then, according to the large value of the dielectric constant in $\mathrm{SrTiO}_{3} / 6 /$, it may be assumed a very small activation energy when compared to $k_{B} T$ and hence the charge carriers arising from the Nbshallow donors will be in the conduction band. This situation seems to be preserved in the whole temperature range investigated here for sample $B$, the shallow donors introduced by the reduction being predominant. For a smaller reduction degree (sample A),

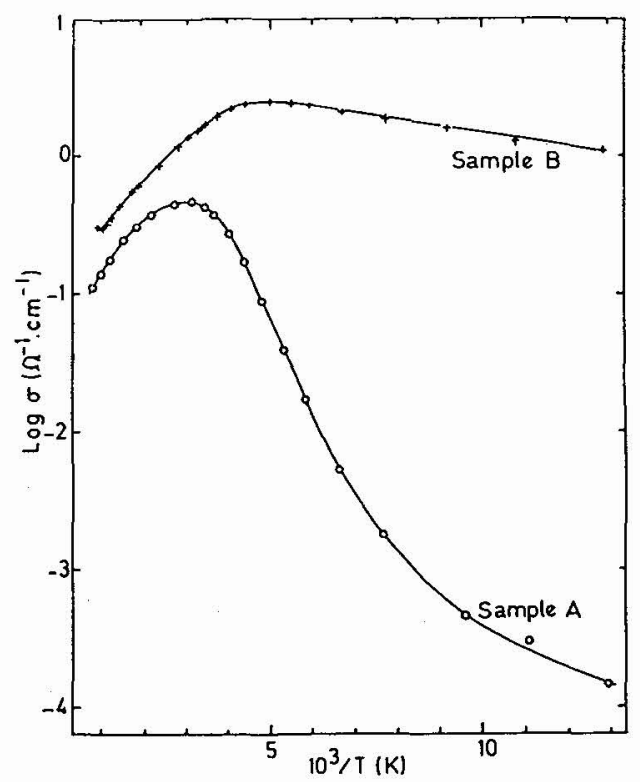

Fig. 2 - Variation of the electrical conductivity versus temperature. 
at low temperature, the conductivity is thermal1y activated and a situation of acceptor-donor compensation predominates. Indeed, compensating centers may originate from either impurity ions, such as manganese-II ions detected by EPR analysis, or nonstoichiometric defects, such as vacancies $/ 1 /$.

Thermoelectric power measurements were also performed in the same temperature range (Fig. 3) on samples. A and B. For the highest temperatures, corresponding to the metallic-type regime, the Seebeck coefficient of both samples $A$ and $B$ is constant and reaches a high negative value of about $-700 \mu \mathrm{V} \mathrm{K}^{-1}$

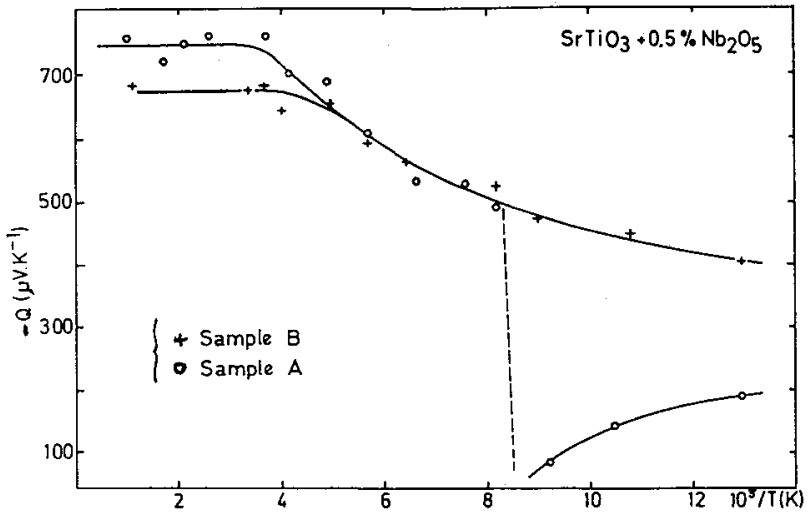

Fig. 3 - Temperature dependence of the Seebeck coefficient.

At lower temperature the absolute value of the Seebeck coefficient decreases with temperature. These results are in agreement with those reported by Frederikse et al $/ 2 /$ who also shown, by combining thermoelectric power and Hall mobility measurements, an important decrease of the effective mass from $16 \mathrm{~m}_{0}$ at room temperature to $6 \mathrm{~m}_{0}$ at $78 \mathrm{~K}$ that indicates the negligible contribution of phonon-drag to Seebeck coefficient $/ 2,7 /$. In the case of the 1 ightly reduced specimen (A) that is the most compensated, an important break is observed in the variation of the thermoelectric power at about $110 \mathrm{~K}$ which is the temperature of the tetragonal to cubic transition for strontium titanate. This transition was not noted by Frederikse at al /2/, who only reported anomalies in the vicinity of $40 \mathrm{~K}$, temperature of the ferroelectric transition.

\section{IV - INFRARED SPECTRA}

The reflection spectrum of reduced niobium doped strontium titanate (sample B) at $80 \mathrm{~K}$ is shown in Fig. 4 .

It is fitted on the basis of the four-parameter model /4/ which assumes the following factorized form of the dielectric function:

$$
\varepsilon=\varepsilon_{\infty} \pi \frac{\Omega_{j L O}^{2}-\omega^{2}+i \gamma_{j L O} \omega}{\Omega_{j T O}-\omega^{2}+i \gamma_{j T O} \omega}
$$


where $\Omega_{j}$ and $\gamma_{j}$ denote the frequencies and the dampings of longitudinal optical (Lo) and transverse optical (TO) vibrational modes respectively. The value of $\varepsilon_{\infty}$ is derived from the reflection spectrum by using the Fresnel formula /4/. This spectrum is quite similar to that of a single crystal having the same composition. The imaginary part of the dielectric (TO modes) and inverse dielectric (LO modes) functions calculated according to Eq. (1) is shown in Fig. 4 by solid lines. The three specific modes of strontium titanate may be observed on both the To and Lo phonon spectra. A LO phonon mode, which is not noticeable in Fig. 4, exists at about $175 \mathrm{~cm}^{-1}$. It may be observed when the spectrum is magnified by about a factor 50 .

The presence of a coupled plasmon mode may be considered as this is the case in most semi-conductors with large electron concentration. According to the mode1 developed for the study of the LO phonon-plasmon coupling in nonstoichiometric rutile single crystals $/ 8 /$, the decoupling of the LO phonon and plasmon modes was achieved by fitting the IR reflection spectrum with the following dielectric function :

$$
\varepsilon / \varepsilon_{\infty}=\prod_{j} \frac{\Omega_{j L O}^{\prime 2}-\omega^{2}+i \gamma_{j L O}^{\prime} \omega}{\Omega_{j T O}^{2}-\omega^{2}+i \gamma_{j T O} \omega}-\frac{\Omega_{p}^{2}+i\left(\gamma_{p}-\gamma_{0}\right) \omega}{\omega\left(\omega-i \gamma_{0}\right)}
$$

where the second term reduces to a Drude expression when the plasmon damping $\gamma_{p}$ measured near the plasma frequency $\Omega_{p}$ is equal to the static value $\gamma_{0}$. The parameter $\Omega_{j L O}^{\prime}$ and $\gamma_{j L O}^{\prime}$ represent the decoupled LO modes. The TO and LO components of the plasmon response alone deduced from the fit with the model Eq. (2) are shown in Fig. 4 as dashed lines. It may be noted that the sintered polycrystalline sample of strontium titanate is characterized by a plasma frequency equal to $275 \mathrm{~cm}^{-1}$ about at $80 \mathrm{~K}$. This value is in agreement with the results obtained on $\mathrm{Nb}^{-\mathrm{SrTiO}_{3}}$ single crystals with various compositions.

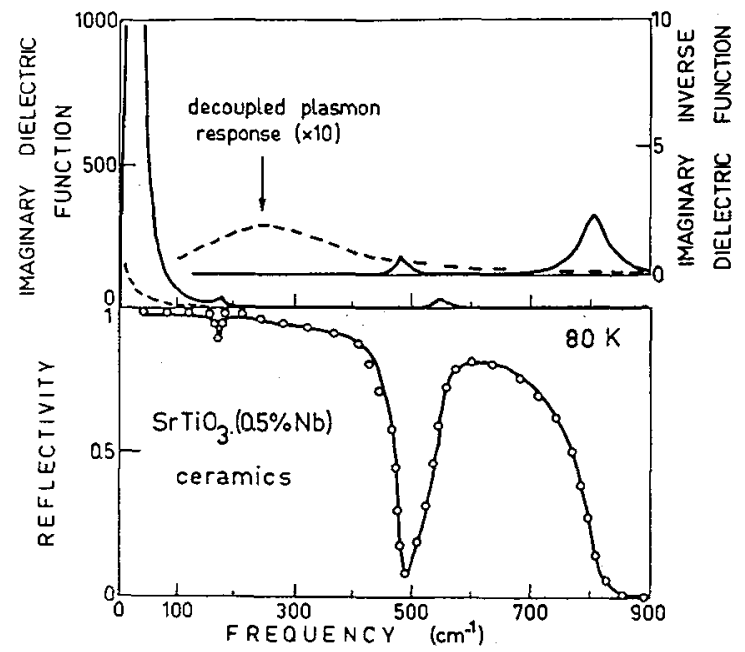

Fig. 4 - Spectral analysis of sample B at $80 \mathrm{k}$. Solid lines are calculated on the basis of the model Eq. (1) - (o) denotes experimental data. 
Polycrystalline Nb-doped strontium titanate has been prepared by using an organometallic route for the powder synthesis. The electrical properties of 0.5 mo $1 \%$ doped samples reveal a metallic-type behaviour at high temperature while at lower temperature an acceptor-donor compensation situation is observed for the lightly reduced sample. The latter exhibits a thermally activated conductivity and presents a noticeable anomaly in the temperature dependence of the Seebeck coefficient at about $110 \mathrm{~K}$.

The IR reflection spectra are quite similar to those obtained with single crystals. Then, the same models have been used for the fitting of the dielectric function and the decoupling of the LO phonon-plasmon modes.

\section{REFERENCES}

/1/ Chan, N. H., Sharma, R. K. and Smyth, D. M., J. Electrochem. Soc. 128 (1981) 1762 .

12/ Frederikse, H. P. R., Thurber, W. R. and Hosler, W. R., Phys. Rev. A 134 (1964) 442.

13/ Tufte, 0. N. and Chapman, P. W., Phys. Rev. 155 (1967) 155.

14/ Gervais, F.,"Infrared and millimeter waves", Vol.III, Ed. Button, K. J., Academi.c Press, New York (1983) 279.

15\% Pechini, M., U.S. Patent 3330697 (1967).

16/ Weaver, H. E., J. Phys. Chem. Solids 11 (1959) 274.

17/ Conyers Herring, Phys. Rev. A 96 (1954) 1163.

/8/ Baumard, J. F. and Gervais, F., Phys. Rev. B 15 (1977) 2316. 